\title{
An Extremely Rare Case of the Traumatic Spinal Epidural Hematoma in a Child: Case Report and Review of the Literature
}

Ivan Domazet*, Ivan Pasalic, Jakob Nemir, Hrvoje Jednacak and Miroslav Vukic

Department of Neurosurgery, University Hospital Centre Zagreb, Croatia

*Corresponding author: Ivan Domazet, Department of Neurosurgery, University Hospital Centre Zagreb, Croatia, Kispaticeva 12, 10000 Zagreb, Croatia

Submission: December 12, 2017: Published: January 22, 2018

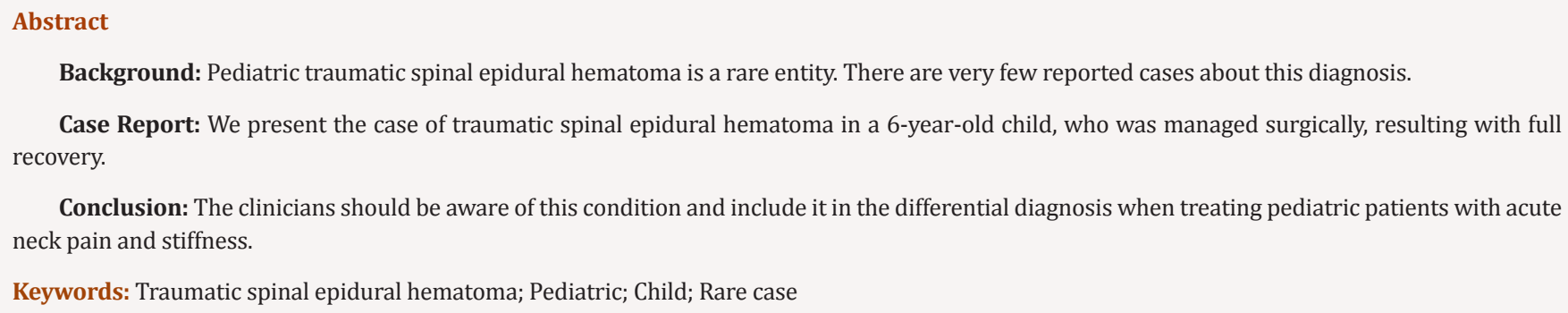

Conclusion: The clinicians should be aware of this condition and include it in the differential diagnosis when treating pediatric patients with acute neck pain and stiffness.

Keywords: Traumatic spinal epidural hematoma; Pediatric; Child; Rare case

\section{Introduction}

Spinal epidural hematoma in pediatric population is a very rare condition, and until today 40 cases were reported, but only 6 of them were traumatic [1]. We present the case of 6-year-old girl Case Report with traumatic spinal epdiural hematoma extending from $\mathrm{C} 2$ to $\mathrm{C} 4$ vertebral levels, who was operated in our Pediatric Neurosurgical Unit.
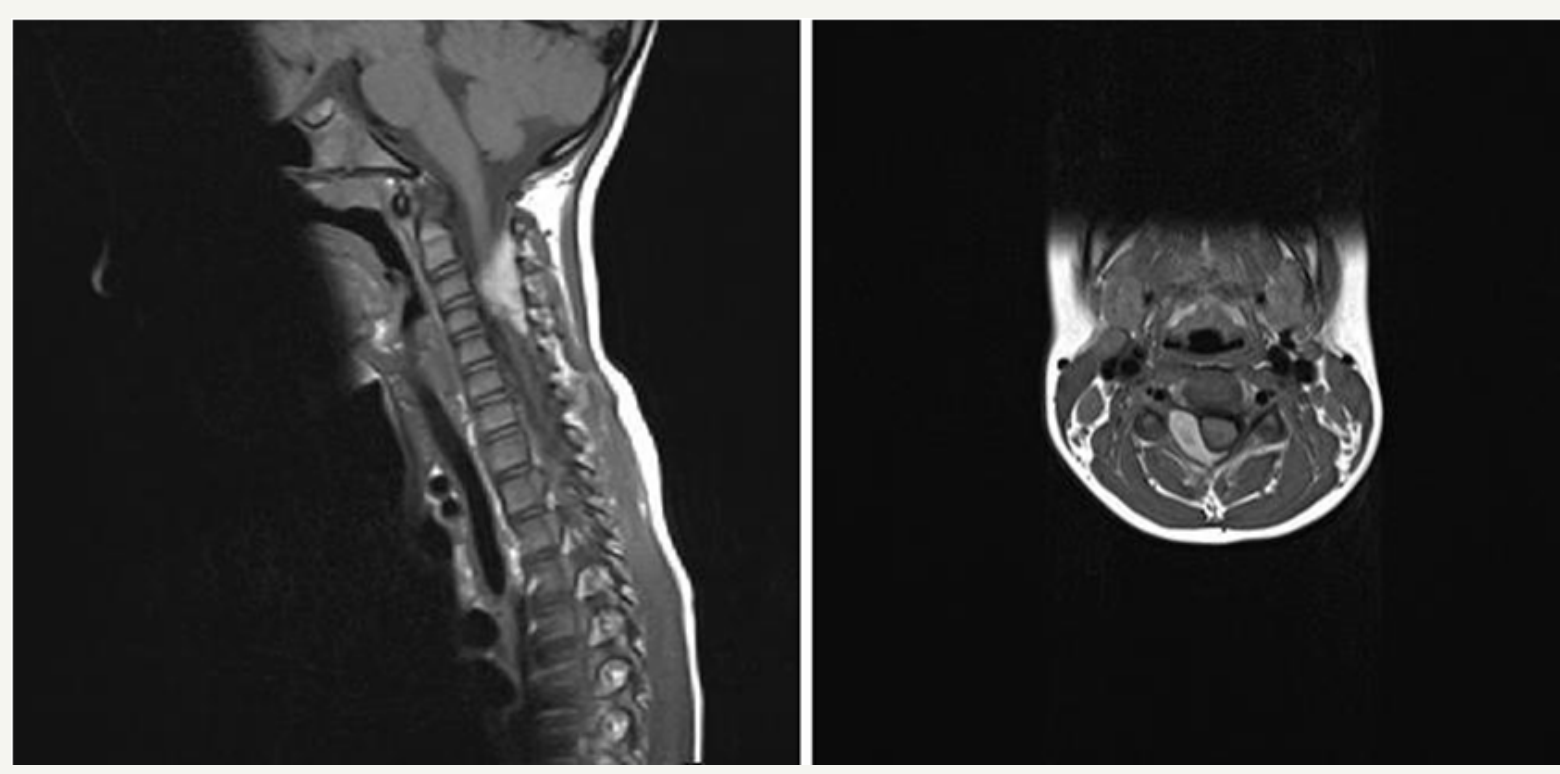

Figure 1: Preoperative MRI scan. 
A 6-year old girl was admitted to Pediatric Neurosurgical Unit presenting with severe neck pain, stiffness and decrease in neck movement. She did not have any weakness in upper or lower limbs. Her symptoms started ten days earlier when she received a punch in the neck while she was playing with other children in her kindergarten. Her mother took her to the chiropractic but the symptoms sustained. After the hospitalization hematological profile was analysed and the results were within normal limits (platelet count 333×109/L, prothrombin time 0.73, international normalized ratio 1.15, partial thromboplastin time 29 seconds, fibrinogen $2.1 \mathrm{~g} / \mathrm{L}$ ). The MRI scan of the cervical spine showed epidural hematoma with spinal cord compression extending from C2 to C4 vertebral levels (Figure 1). Emergency operation was indicated. Surgery was performed in prone position. Midline vertical skin incision extending from $\mathrm{C} 2$ to $\mathrm{C} 5$ levels was made and right-sided C4 hemilaminectomy and evacuation of the underlying epidural hematoma were performed. No masses or vascular anomalies were detected during the operation. Skin was suttured and the patient was postoperatively transferred to the Pediatric Neurosurgical Unit. Histopathological diagnosis was: hematoma. She was released from the hospital on the fifth postoperative day. At the moment of the release she was neurologically intact with recuperated neck movement and no neck pain. The MRI scan that was performed 8 weeks after the surgery demonstrated normal postoperative findings with good morphology of the spinal canal and its structures and without signs of the residual epidural collection (Figure 2). During the follow up in the outpatient clinic she was asymptomatic and neurologically intact.

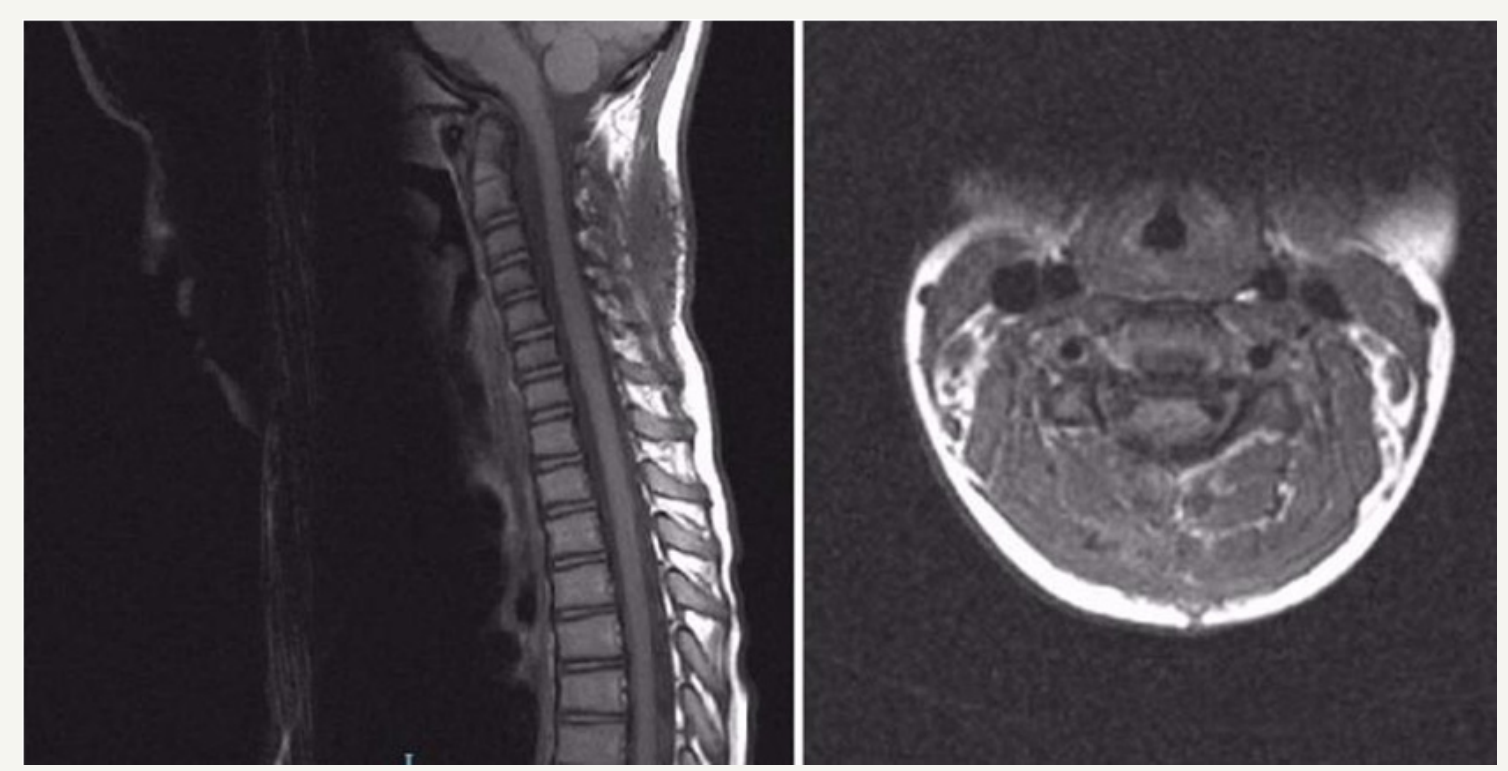

Figure 2: Postoperative MRI scan.

\section{Discussion}

Non-traumatic spinal epidural hematomas may be caused by hematologic diseases (lymphoma, leukemia), tumors, arteriovenous malformations, vasculitis, coagulopathies, infections, antiocoagulants, hypertension and ankylosing spondilitis [2,3]. Jackson was the first who reported about spontaneous spinal epidural hematoma in 1869 [2,4,5]. Spinal epidural hematomas in pediatric patients are rare entity. The majority of these cases have spontaneous etiology, and spinal epidural hematomas with traumatic etiology are extremely rare [1-3,5]. Rangarajan et al. [1] reported that so far only 40 cases of pediatric spinal epidural hematomas have been reported in literature, and only 6 of them had traumatic etiology [1]. Fountas et al. [5] reported that annual incidence of spontaneous spinal epidural hematoma has been reported to be 0.1 per 100000 patients in general population, but even lower in pediatric population [5]. Simptoms are caused by spinal cord and spinal root compression and patients are usually presented with neck pain, stiffness, decrease in neck movement, irritability, crying, hemiparesis, paraparesis, quadriparesis and even paraplegia [1-3,5]. MRI imaging should be used as a diagnostic technique of choice for the verification of the spinal epidural hematoma [6-8]. MRI angiography, CT and CT angiography can be used as a supplement for MRI imaging. In most cases, after MRI imaging was performed, location of the spinal epidural hematoma is verified in cervical region, as it was in our case [1-3,5], but there are cases that reported hematomas in thoracic and lumbar region [1-3,5,9]. Lim et al. [3] reported that an average duration of the symptoms prior to surgery was 7.1 days (range 2-14 days) [2]. In two reported cases of the traumatic epidural spinal hematomas patient was admitted to hospital few hours after the trauma, and in one case patient had a trauma 14 days before the admission $[1,2,5]$. In our case patient was admitted to our unit ten days after the trauma, and she was operated on the very same day. Lim et al. [3] reported about 20-month-old girl with spontaneous spinal epidural hematoma extending from C7-T4 who was treated with two-level laminotomy [3]. They also published review of literature in which they recommended rapid surgical evacuation as a treatment of choice for the symptomatic spontaneous spinal epidural hematomas [3]. Rangarajan et al. [1] reported about 8-month-male infant with traumatic epidural spinal hematoma in region C4-T1 
who was treated with C6 and C7 laminoplasty and the evacuation of the hematoma [1]. Gupta et al. [2] reported about six-year-old boy with the traumatic spinal epidural hematoma in the region C3-T4 who was treated with $\mathrm{C} 7$ hemilaminectomy and the evacuation of the hematoma [2]. Fountas et al. [5] published case report about 12year old girl with traumatic spinal epidural hematoma extending from C3 to C7, and also published review of literature in which they recommended hemilaminectomy as a treatment of choice because it provides adequate exposure for the evacuation of hematoma and it minimizes the risk of postlaminectomy deformity $[5,10]$. In our case unilateral hemilaminectomy was selected as a treatment of choice because it provided good exposure of the hematoma, surgeons were able to evacuate whole hematoma, and we believe it will not affect the range of motion of the cervical spine in the future.

\section{Conclusion}

Traumatic spinal epidural hematoma in pediatric population is an extremely rare condition. Although clinical simptoms of the traumatic spinal epidural hematomas are not very specific, early recognition of this condition and surgical intervention are most important for the good outcome. The clinicians should be aware of this condition and include it in the differential diagnosis when treating pediatric patients with acute neck pain and stiffness.

\section{References}

1. Rangarajan V, Mavani SB, Nadkarni TD, Goel AH (2013) Traumatic cervical epidural hematoma in an infant. J Craniovertebr Junction Spine $4(1): 37-39$.
2. Gupta V, Kundra S, Chaudhary AK, Kaushal RK (2012) Cervical epidural hematoma in a child. J Neurosci Rural Pract 3(2): 217-218.

3. Lim JJ, Yoon SH, Cho KH, Kim SH (2008) Spontaneous spinal epidural hematoma in an infant: a case report and review of the literature. J Korean Neurosurgic Soc 44(2): 84-87.

4. Alva NS (2000) Traumatic spinal epidural hematoma of a 10-month-old male: a clinical note. Pediatr Neurol 23(1): 88-89.

5. Fountas KN, Kapsalaki EZ, Robinson JS (2006) Cervical epidural hematoma in children: A rare clinical entity. Case report and review of the literature. Neurosurg Focus 20(2): E6.

6. Pan G, Kulkarni M, MacDougall DJ, Miner ME (1988) Traumatic epidural hematoma of the cervical spine: diagnosis with magnetic resonance imaging. Case report. J Neurosurg 68(5): 798-801.

7. Bennett DL, George MJ, Ohashi K, El-Khoury GY, Lucas JJ, et al. (2005) Acute traumatic spinal epidural hematoma: imaging and neurological outcome. Emerg Radiol 11(3): 136-144.

8. Chang FC, Lirng JF, Luo CB, Yen YS, Guo WY, Teng MM, et al. (2005) Evaluation of clinical and MR findings for the prognosis of spinal epidural haematomas. Clin Radiol 60(7): 762-770.

9. Sumner DW (1962) Spontaneous spinal extradural hemorrhage due to hemophilia. Report of a case. Neurology 12: 501-502.

10. Yasuoka S, Peterson HA, MacCarthy CS (1982) Incidence of spinal column deformity after multilevel laminectomy in children and adults. J Neurosurg 57(4): 441-445. 